\title{
Aplicação do overall equipment effectiveness no sistema produtivo de uma vinícola
}

\section{Application of overall equipment effectiveness in the productive system of a winery}

\author{
Pedro Vieira Souza Santos Engenheiro de Produção. Universidade Federal do Vale do São Francisco (UNIVASF) - Brasil. \\ pedrovieirass@hotmail.com.
}

\section{RESUMO}

O termo eficiência, de forma geral, retrata à medida que um resultado é produzido com menor quantidade observada de desperdício e esforço, isto é, realizar uma operação com o mínimo de perdas. Diante disso, a verificação da eficiência dos equipamentos do processo pode auxiliar na redução de problemas complexos ligados a produção, intervindo ainda na análise do sistema produtivo. Assim, este artigo teve como objetivo aplicar o Overall Equipment Effectiveness (OEE) em um sistema produtivo, para monitorar e avaliar a situação dos equipamentos utilizados no processo, evidenciando as possíveis melhorias. $O$ presente estudo, do ponto de vista metodológico, classificou-se como um estudo de caso, realizado em uma empresa do setor vinícola, localizada na Região Administrativa Integrada de Desenvolvimento (RIDE) em Pernambuco. $O$ estudo, pôde apontar que os equipamentos escolhidos para análise, a prensa $(\mathrm{OEE}=83,47 \%)$ e as duas máquinas de envase $(69,19 \%$ e $69,38 \%$, respectivamente), podem ter seu desempenho melhorado. Para isto, o plano de ação comporta-se como uma ferramenta gerencial fundamental e deve ter sua execução para que a empresa tenha, de fato, mais lucro e melhor qualidade nos produtos fabricados. Como principal limitação do estudo, pode-se citar a quantidade de equipamentos analisados, tendo em vista que a empresa conta com uma vasta combinação destes. Além disso, a verificação dos valores foi executada em apenas um mês, recomendandose, portanto, para estudos futuros, a análise e comparação dos valores por mês.

Palavras chave: Gestão da produção. Desempenho. Melhoria Contínua.

\begin{abstract}
The term efficiency, in general, refers to a result produced with the least observed amount of waste and effort, that is, performing an operation with minimal loss. Therefore, checking the efficiency of the equipment involved in a given process can help to reduce complex problems related to production, also intervening in the analysis of the production system. Thus, this article aimed to apply Overall Equipment Effectiveness (OEE) in a production system to monitor and evaluate the situation of the equipment used in the process, highlighting the possible improvements. From the methodological point of view, the present study was classified as a case study, conducted in a company in the wine sector, located in the Integrated Administrative Development Region (RIDE) in Pernambuco. The study showed that the equipment chosen for analysis, the press $(\mathrm{OEE}=83.47 \%)$ and the two filling machines $(69.19 \%$ and $69.38 \%$, respectively), can have their performance improved. For this purpose, the action plan functions as a fundamental managerial tool and must have its execution so that the company actually achieves more profit and better quality in the manufactured products. As a major limitation of the study, we can cite the amount of equipment analyzed, given that the company has a wide combination of these. In addition, the verification of the values was performed in only one month, therefore, for future studies, the analysis and comparison of the values per month is recommended.
\end{abstract}

Keywords: Production management. Performance. Continuous Improvement. 


\section{INTRODUÇÃO}

Frente a um ambiente cada vez mais competitivo, as empresas tendem a aprimorarem suas competências internas na busca por sustentabilidade dos negócios. Logo, alguns fatores são essenciais, como a eficiência das operações, a qualidade do processo e o atendimento adequado das demandas dos clientes, exemplos que norteiam a gestão no acompanhamento das mudanças que ocorrem no mercado (MAŠlć; DŽUNIĆ; NEŠIĆ, 2014; SANTOS; SANTOS, 2018). Para Reeves e Deimler (2011) a organização deve, de fato, aprimorar seus processos para garantia de vantagem competitiva.

Wudhikarn (2013) afirma que, perante o acirrado cenário no qual as empresas encontram-se, estas devem reduzir seus custos de manufatura, além de manutenção da qualidade dos itens produzidos, observando a sua eficiência. Garza-Reyes et al. (2010) e Santos e Araújo (2018) afirmam que o termo eficiência, de forma geral, retrata à medida que um resultado é produzido com menor quantidade observada de desperdício e esforço, isto é, realizar uma operação com o mínimo de perdas. Singh et al. (2013) complementa ainda ao afirmar que, diversas técnicas foram desenvolvidas ao longo do tempo para melhoria na gerência dos processos. Para os autores, a verificação da eficiência dos equipamentos do processo pode auxiliar na redução de problemas complexos ligados à produção, intervindo ainda na análise do sistema produtivo como um todo.

Segundo Aman et al. (2017), uma forma de verificar a eficiência dos equipamentos industriais é a partir do Overall Equipment Effectiveness (OEE). De acordo com Mourtzis e Doukas (2012) e Santos (2018), traduz-se como o indicador geral que analisa a eficácia considerando três termos de medida: Disponibilidade, Desempenho e Qualidade. Por meio do OEE, a avaliação é comparada com o potencial de funcionamento da máquina, fundamentando-se na ideia de que esta capacidade pode ser comprometida por perdas.

O indicador OEE faz parte do programa de Manutenção Produtiva Total (TPM). Trata-se de uma metodologia de gestão que pode identificar perdas existentes no processo produtivo, maximizando a utilização do ativo industrial e, ainda, garantindo a manufatura de alta qualidade (KRYNKE; KNOP; MIELCZAREK, 2014). Afefy (2013) comenta que o objetivo do sistema japonês TPM é reduzir as perdas que afetam o funcionamento pleno dos equipamentos ao nível máximo possível.

Assim, este artigo tem como objetivo aplicar o Overall Equipment Effectiveness (OEE) no sistema produtivo de uma vinícola localizada no Vale do São Francisco, em Pernambuco, para monitorar e avaliar a situação dos equipamentos utilizados no processo, evidenciando as possíveis melhorias. A aplicação do indicador em uma empresa de vinícola, justifica-se pelo fato de que na Região há grande concentração de empresas desse ramo, o que gera oportunidade de intervir no processo, por meio de estudos como esse, para assim analisar a eficiência do mesmo e contribuir para a melhor gestão organizacional.

\section{ABORDAGEM TEÓRICA}

Nos subtópicos a seguir serão apresentados os principais conceitos ligados ao tema de Manutenção Produtiva Total (TPM), além da caracterização dos indicadores do Overall Equipment Effectiveness (OEE).

\subsection{Total Productive Maintenance - TPM}

O termo Total Productive Maintenance, foi introduzido e desenvolvido pelos japoneses em meados de 1971, oriundo de demandas por soluções diante dos problemas de manutenção e suporte em ambientes fabris. Embora originado no Japão, Sivanantham e Sivaram (2017) citam que o TPM é amplamente aceito e implementado com sucesso em todo o mundo, reflexo da percepção de que a técnica é importante e viável. A princípio, baseia-se na disposição de equipes para manutenção, preventiva e/ou produtiva, envolvendo todos os níveis da empresa, desde o executivo ao operador. O método foi provado ser bem-sucedido por ajudar a aumentar a produtividade e, em geral, a eficácia do equipamento (KATKAMWAR; WADATKAR; PAROPATE, 2013).

Segundo Takahashi e Osada (2000), o termo Total Productive Maintenance (TPM), traduzido para língua portuguesa como Manutenção Produtiva Total, reporta-se a uma metodologia que abrange toda a organização, com todos os colaboradores em participação. Pode ser entendida como uma filosofia de gestão 
direcionada para o equipamento. O objetivo do programa TPM, de acordo com Singh et al. (2013) é melhorar a produtividade do sistema, assim como a qualidade. Comporta-se como uma abordagem inovadora de manutenção que otimiza a eficácia do equipamento, elimina gargalos e promove a manutenção autônoma por parte do operador.

A filosofia do TPM foca nas seis grandes perdas que são diretamente responsáveis pela redução e/ou limitação do rendimento operacional geral dos equipamentos, são elas: perda por quebras, perda por alteração de linha, perda por operação em vazio e ainda pequenas paradas, perda por velocidade reduzida em relação à nominal, perda por defeitos de produção e perda por queda de rendimento (KARDEC; NASCIF, 2009). Além disso, Manutenção Produtiva Total conta com oito pilares, conforme Figura 1, que de acordo com Adesta, Prabowo e Agusman (2018), funcionam como um sistema para maximizar a eficácia da produção de qualquer indústria. Cada um destes pilares é resumido a partir do Quadro 1.

Figura 1 - Pilares do TPM

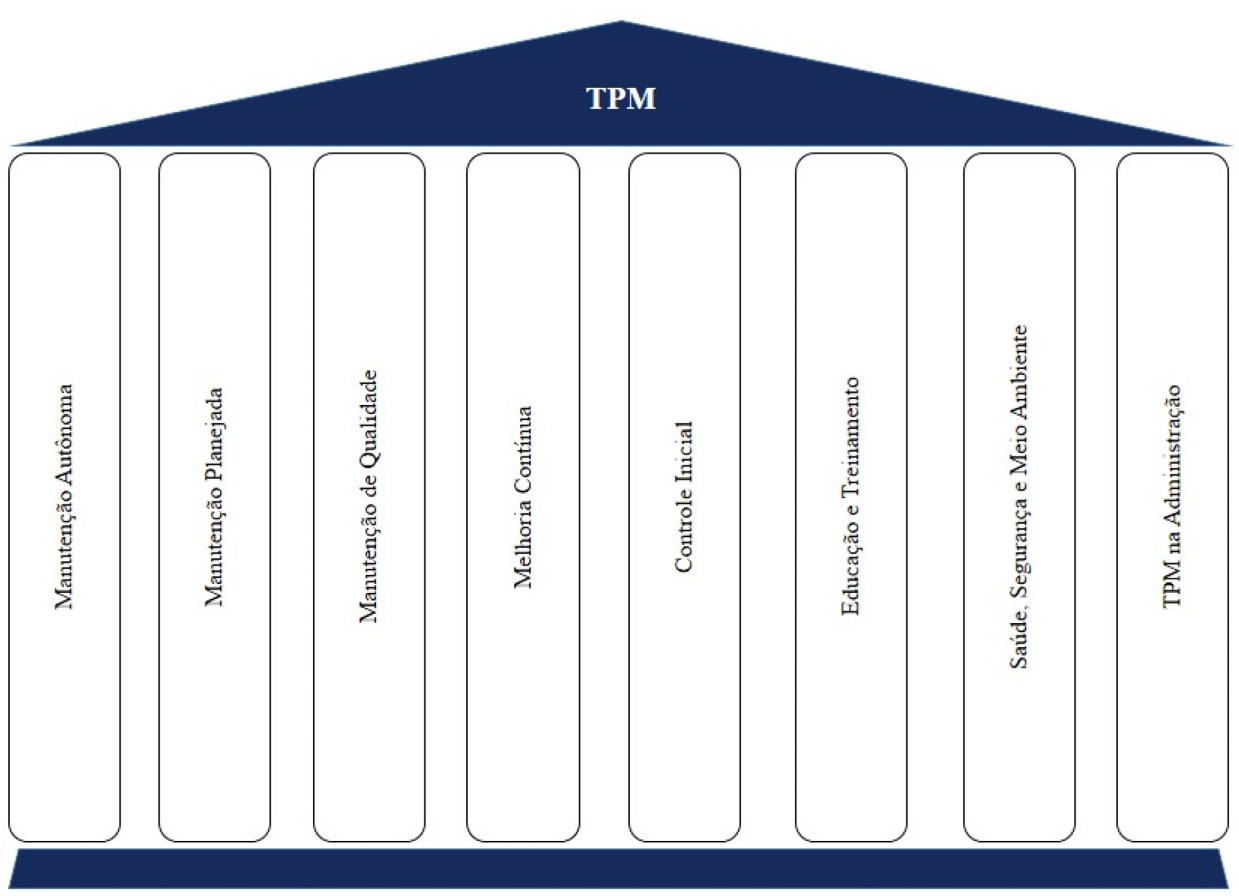

Fonte: Adaptado de Pandey e Raut (2016).

Quadro 1 - Os 8 pilares do TPM

\begin{tabular}{|c|c|}
\hline Pilar & Descrição \\
\hline Manutenção autônoma & $\begin{array}{c}\text { Operadores de equipamentos responsáveis para executar a } \\
\text { manutenção básica de equipamentos locais. }\end{array}$ \\
\hline $\begin{array}{l}\text { Manutenção planejada } \\
\text { (Keikaku-Hozen) }\end{array}$ & $\begin{array}{l}\text { Manutenção programada usando o histórico taxa de falha do } \\
\text { equipamento. }\end{array}$ \\
\hline Manutenção de Qualidade & Qualidade intrínseca no equipamento para reduzir defeitos. \\
\hline $\begin{array}{l}\text { Melhoria contínua } \\
\text { (Kaizen) }\end{array}$ & Uso de equipes multifuncionais para atividades de melhoria. \\
\hline Controle Inicial & Minimizar o tempo de introdução do produto e processo. \\
\hline Educação e Treinamento & $\begin{array}{l}\text { Preenchimento da lacuna de habilidades e conhecimento através da } \\
\text { formação continuada de todos os trabalhadores. }\end{array}$ \\
\hline $\begin{array}{l}\text { Saúde, Segurança e } \\
\text { Meio Ambiente }\end{array}$ & Ambiente de trabalho ideal sem acidentes e organizado. \\
\hline TPM na administração & $\begin{array}{l}\text { Propagação dos princípios para a administração ou funções dentro de } \\
\text { uma organização. }\end{array}$ \\
\hline
\end{tabular}

Fonte: Adaptado de Pandey e Raut (2016) 
Na ótica de Jeong e Phillips (2001), as iniciativas de TPM abordam as principais perdas e desperdícios relevantes para o sistema de produção. Dal, Tugwell e Greatbanks (2000) complementam ainda ao afirmarem que, a filosofia estabelece então uma avaliação sistemática e contínua das instalações industriais, direcionando a gestão do desempenho como medida fundamental para eficiência global. Isto posto, fica evidente que a implementação da filosofia TPM pode acarretar em uma maior qualidade em todas as faces das operações de manufatura (CHEN et al., 2015). Com isso, Chong, Chin e Hamzah (2015) comentam que a prática reúne atividades que, quando aplicadas em processos de produção, otimizam a eficiência do equipamento e seu rendimento.

Portanto, traduz-se basicamente como um conjunto de atividades organizadas que são capazes de melhorar gestão da planta industrial, podendo ser realizada individualmente e com a ajuda de equipes (PARIKH; MAHAMUNI, 2015). Umas das técnicas utilizadas com base nesse pensamento é o cálculo da eficiência geral do equipamento (OEE).

\subsection{Overall Equipment Effectiveness - OEE}

Desenvolvido em meados dos anos 90, o Overall Equipment Effectiveness tornou-se uma ferramenta de gerenciamento aceita para medir e avaliar a produtividade no chão de fábrica (MARKUS; EBERHARD, 2015). A eficácia geral do equipamento, OEE, é uma maneira de monitorar e melhorar a eficiência do processo, a partir da avaliação da eficiência dos equipamentos, dividido em três métricas: disponibilidade, desempenho e qualidade (AMAN et al., 2017). Bariani e Del'Arco Júnior (2006) em concordância com Antunes et al. (2008), definem tais parâmetros como:

Disponibilidade $(\mu 1)$ : quantidade de tempo em que um equipamento ou máquina esteve disponível para operar, comparado com a quantidade programada; se apresentar um baixo valor, indica a ocorrência de diversas paradas durante a produção. É calculado, de acordo com segundo Corrêa e Corrêa (2004), seguindo a equação:

$$
\begin{gathered}
\mu 1=(\mathrm{TPP}-\mathrm{TPnP}) / \mathrm{TPP} \\
\text { Onde: } \\
\mu 1=\text { Índice de disponibilidade } \\
\mathrm{TPP}=\text { Tempo de produção planejado } \\
\mathrm{TPnP}=\text { Tempo de paradas não planejadas }
\end{gathered}
$$

Desempenho $(\mu 2)$ : quanto o equipamento trabalha em relação ao tempo de ciclo ideal para produção de uma peça; ainda considera perdas de ritmo ocorridas por problemas diversos como a redução de velocidade, paradas rápidas e operações em vazio. Matematicamente, segundo Corrêa e Corrêa (2004), é obtido por meio da equação abaixo:

$$
\begin{gathered}
\mu 2=(\mathrm{TCU}-\mathrm{QPP}) / \mathrm{TP} \\
\text { Onde: } \\
\mu 2=\text { Índice de desempenho } \\
\mathrm{TCU}=\text { Tempo de ciclo por unidade } \\
\mathrm{QPP}=\text { Quantidade de produtos processados } \\
\mathrm{TP}=\text { Tempo em produção }
\end{gathered}
$$

Qualidade $(\mu 3)$ : representa o número total de peças produzidas em conformidade, comparado com o número total de peças produzidas; resultados abaixo do índice de qualidade são identificados quando há ajustes, originando retrabalho e refugos após uma operação de setup, por exemplo. Pode ser definido pela fórmula matemática, segundo Corrêa e Corrêa (2004): 


$$
\begin{gathered}
\mu 3=(\mathrm{QPP}-\mathrm{QPR}) / \mathrm{QPP} \\
\text { Onde: } \\
\mu 3=\text { Índice de qualidade } \\
\mathrm{QPP}=\text { Quantidade de produtos processados } \\
\mathrm{QPR}=\text { Quantidade de produtos de refugo }
\end{gathered}
$$

Por outro lado, Antunes et al. (2008) definem matematicamente o OEE como o resultado do produto entre os três parâmetros, sendo:

$$
\mu \text { geral }=\mu 1 \times \mu 2 \times \mu 3
$$

As pontuações do OEE indicam se o equipamento que está funcionando de forma adequada ou não, na perspectiva de produzir uma peça reduzindo as perdas durante o processo de produção (MADANHIRE; MBOHWA, 2015). O padrão aceitável de classe mundial de OEE é de $85 \%$, com uma taxa de pontuação de disponibilidade de noventa por cento (90\%), além da taxa de pontuação de eficiência de desempenho de noventa e cinco por cento (95\%) e índice de qualidade de 99,9\%, seria ideal na prática (GUPTA; GARG, 2012). Por outro lado, Hansen (2006) aborda ainda métricas nas quais podem ser levadas em consideração no momento de comparar os valores obtidos na prática com estimativas toleráveis, conforme descritas na Tabela 1.

Tabela 1 - Parâmetros de aceitabilidade para $\mu$

\begin{tabular}{cc}
\hline Parâmetro & Critério \\
\hline Abaixo de 65\% & Não aceitável \\
Entre 65\% e $75 \%$ & Bom \\
Entre $75 \%$ e $85 \%$ & Muito Bom \\
\hline
\end{tabular}

Fonte: Adptado de Hansen (2006)

Logo, o OEE pode ser introduzido, como ferramenta estratégica em unidades industriais. Além de otimização da produtividade local, com o indicador é possível maximizar a performance do maquinário utilizado nas etapas do sistema produtivo, melhorando a capacidade de produção (CHARAF; DING, 2015).

Por outro lado, o índice OEE representa um instrumento eficaz na realização de diagnósticos, comparando unidades e/ou componentes do sistema de produção. Ademais, o resultado confiável produzido pelo OEE pode ser usado para propor uma ação corretiva adequada (MAIDEEN et al., 2016). No entanto, com a utilização periódica, o uso do indicador é transformado em um sistema que analisa os dados de produção, identifica possíveis áreas de melhoria e apoia iniciativas enxutas (YUNIAWAN; ITO; BIN, 2013).

\section{ASPECTOS METODOLÓGICOS}

O presente estudo, do ponto de vista metodológico, classifica-se como um estudo de caso. Segundo Yin (2001, p. 32), "o estudo de caso é uma investigação empírica de um fenômeno contemporâneo dentro de um contexto da vida real, sendo que os limites entre o fenômeno e o contexto não estão claramente definido". Logo, através desta pesquisa, é recomendado para desenvolver a teoria de forma indutiva, analisando o caso, aqui relatado, como uma experiência distinta.

A pesquisa foi realizada em uma empresa do setor vinícola, localizada na Região Administrativa Integrada de Desenvolvimento (RIDE) em Pernambuco, no Vale do São Francisco. De acordo com Santos (2017, p. 62), essa região "destaca-se como um grande polo produtor de frutas, voltado tanto à exportação quanto ao mercado interno, mas a produção de vinhos tem também importância não apenas nos aspectos econômico, 
mas também turístico." Tal fato demonstra a importância dos resultados obtidos para garantia de vantagem competitiva para a empresa. Trata-se de uma empresa que atua há 18 anos no setor e que produz diversos produtos, em forma de variedade de vinhos, sucos de uva e outros, na sua planta. Nesta localidade, foram aplicados os conceitos de OEE e suas fórmulas matemáticas para identificação do desempenho global dos equipamentos da indústria.

Assim, a pesquisa comporta-se ainda como explicativa, preocupando-se em identificar os fatores que contribuem para a ocorrência dos fenômenos (GIL, 2007). Isto é, o estudo explica o porquê das coisas em função dos resultados encontrados. De acordo com Gil (2007, p. 43), "uma pesquisa explicativa pode ser a continuação de outra descritiva, posto que a identificação de fatores que determinam um fenômeno exige que este esteja suficientemente descrito e detalhado."

\subsection{Das etapas da pesquisa}

Portanto, para atender aos objetivos do estudo, a seguinte sequência de atividades foi executada:

Discussão e apresentação da proposta: nesta fase, foi discutido, junto à administração da empresa, os ganhos que poderiam ser obtidos por meio da aplicação do OEE no processo produtivo local; dúvidas foram sanadas e os objetivos foram aprimorados;

Escolha do processo: como na planta há diversos tipos de produtos em fabricação, com equipamentos distintos por cada item, o processo de fabricação do vinho tipo suave foi definido como mais viável para o estudo, além de ser o que representa maior participação em termos de vendas na empresa;

Entendimento acerca do sistema produtivo: nesta etapa, a partir de informações repassadas pelo enólogo responsável pelo processo, pôde-se entender mais sobre as particularidades do processo de vinificação;

Levantamento de dados: por meio de coleta, no local, os dados necessários para os cálculos dos índices foram anotados e registrados em planilha. Os equipamentos definidos para submissão ao estudo foram: a prensa (equipamento 01), a máquina de envase $n^{\circ} 01$ (equipamento 02) e a máquina de envase $n^{\circ} 02$ (equipamento 03). Esta fase foi executada entre os meses de maio e junho de 2018;

Cálculo do OEE: Por fim, sabendo os dados fundamentais para aplicação da fórmula matemática, os indicadores referentes a disponibilidade $(\mu 1)$, desempenho $(\mu 2)$ e qualidade $(\mu 3)$ foram determinados por equipamento. Ao final, o OEE individual foi estipulado;

Plano de ação: como complemento ao estudo, um plano de ação foi traçado com o intuito de definir atividades que nortearão a gestão da vinícola a aumentar a eficiência dos equipamentos estudados.

\subsection{Do processo produtivo}

Como forma de abordar a realidade do ambiente de estudo, foi feito um mapeamento simplificado do processo produtivo. Logo, as etapas de produção dos vinhos na vinícola estudada, seguem a ordem descrita na Figura 2. 
Figura 2 - Processo produtivo na vinícola
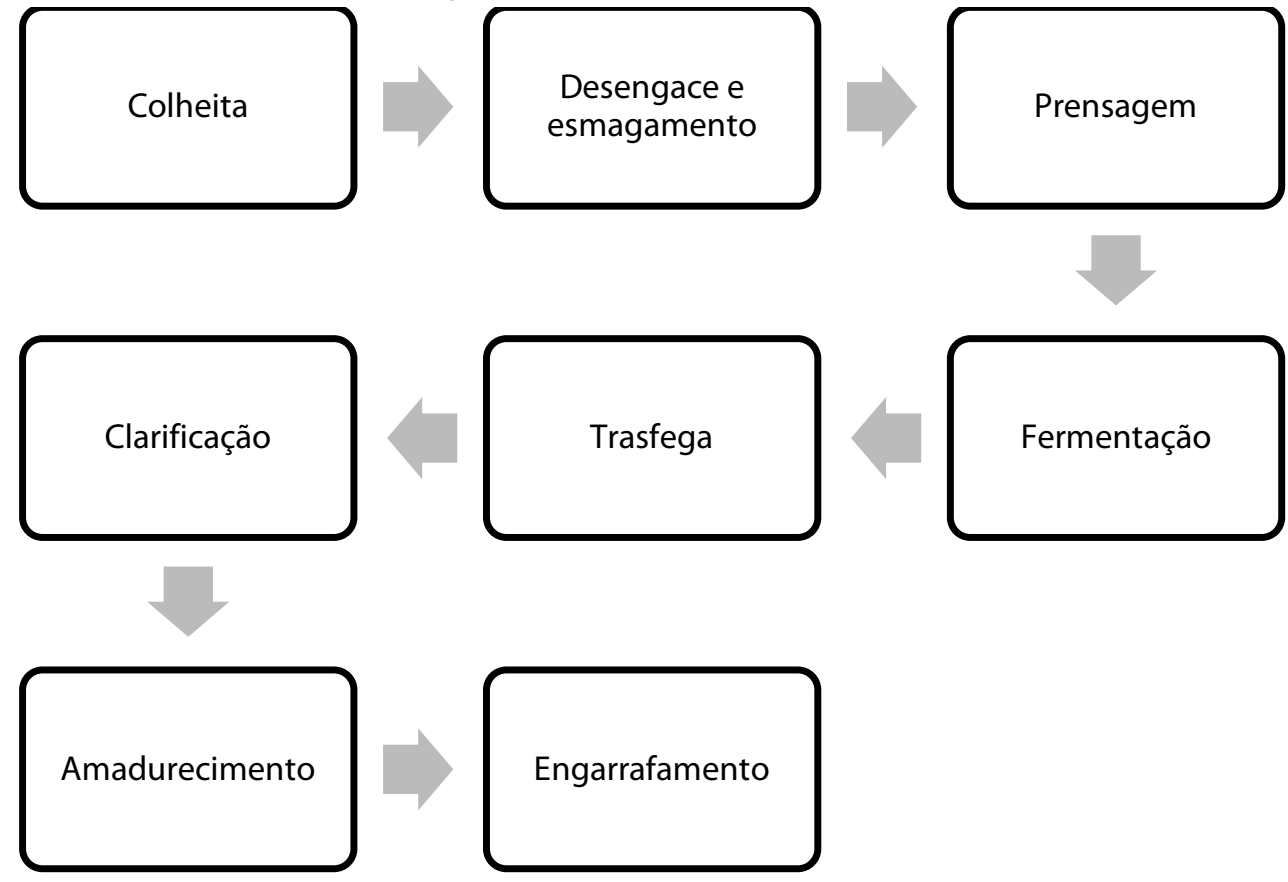

Fonte: Autoria própria (2018).

De forma resumida, cada etapa pode ser descrita, de acordo com o Quadro 2.

Quadro 2 - Descrição sintetizada do processo industrial do vinho

\begin{tabular}{|c|l|}
\hline Etapa & \multicolumn{1}{c|}{ Descrição } \\
\hline Colheita & $\begin{array}{l}\text { Trata-se de um momento importante no processo, que ditará a qualidade } \\
\text { do produto final. É realizada em diferentes épocas do ano, a depender da } \\
\text { variedade da uva e das condições climatológicas da região produtora. }\end{array}$ \\
\hline Desengace e esmagamento & $\begin{array}{l}\text { Nesta fase, as uvas colhidas são introduzidas no equipamento chamado } \\
\text { desengaçadeira e ainda a esmagadeira, que é uma máquina responsável } \\
\text { pela remoção dos engaços (ramificações do cacho de uvas) e ainda } \\
\text { rompimento das cascas das uvas. Assim, o suco da uva pode escorrer, sem } \\
\text { que as cascas e sementes sejam esmagadas. }\end{array}$ \\
\hline Prensagem & $\begin{array}{l}\text { Aqui, o mosto (produto oriundo da etapa anterior) é submetido à } \\
\text { prensa para que haja a separação das cascas e sementes do suco. }\end{array}$ \\
\hline Frasfega & $\begin{array}{l}\text { Por meio dos tanques de aço, as chamadas leveduras se alimentam do } \\
\text { açúcar natural contido no suco das uvas, convertendo-o em álcool e } \\
\text { dióxido de carbono. }\end{array}$ \\
\hline Clarificação & $\begin{array}{l}\text { Nessa operação há a passagem do vinho de um tanque para o outro, } \\
\text { separando o produto inicial de resíduos sólidos, matéria orgânica e ainda } \\
\text { bactérias depositadas no fundo do tanque de aço. }\end{array}$ \\
\hline Engarrafamento & $\begin{array}{l}\text { Trata-se de um processo onde são removidos componentes indesejados } \\
\text { aolíquido. Há uma estabilização do tipo ao frio, ao calor e microbiológica. }\end{array}$ \\
\hline Amadurecimento & $\begin{array}{l}\text { Fase importante e decisiva no resultado qualitativo da bebida. O } \\
\text { processo de amadurecimento é feito em tanques de aço ou barris de } \\
\text { carvalho. Assim algumas características são agregadas ao produto, como } \\
\text { aromas e sabores, deixando-os mais macios. }\end{array}$ \\
\hline $\begin{array}{l}\text { Nessa fase final, o vinho oriundo das etapas passadas, é engarrafado e } \\
\text { deixado de repouso e após segue para comercialização. }\end{array}$ \\
\hline
\end{tabular}

Fonte: Autoria própria (2018). 
Vale salientar que o processo produtivo é mais complexo do que descrito no Quadro 2, dependendo de vários fatores, como tipo de vinho, particularidades do produtor, localização do plantio e outras. Mas, as etapas fundamentais de produção de um vinho são, basicamente as citadas acima.

\section{RESULTADOS E DISCUSSÃO}

Nessa seção serão apresentados os resultados encontrados. Isto é, o cálculo detalhado do índice de eficiência de cada equipamento submetido ao estudo e as respectivas análises à luz da literatura.

\subsection{Avaliação dos equipamentos}

O processo produtivo da empresa funciona em ritmo padrão de $08 \mathrm{~h} 00 \mathrm{~min}$ por dia, sendo $04 \mathrm{~h} 00 \mathrm{~min}$ realizadas no primeiro período, com intervalo de $01 \mathrm{~h} 00 \mathrm{~min}$ para almoço, completando as $04 \mathrm{~h} 00 \mathrm{~min}$ restantes no segundo período do dia.

Como o processo possui diversos equipamentos, foram escolhidos os que mais enquadram-se na relevância e facilidade de coleta de dados. Conforme citado anteriormente na sessão 3.1, os equipamentos estão representados como: prensa (equipamento 01), a máquina de envase no 01 (equipamento 02) e a máquina de envase no 02 (equipamento 03). Salienta-se que, devido a limitação do artigo, os dados coletados não foram citados nas próximas sessões, contudo, apenas o valor necessário foi introduzido por meio das equações. Os valores são representados na forma de média, relativa ao período de 28 dias de coleta, entre maio e junho de 2018.

\subsection{Cálculo dos índices}

O primeiro indicador calculado, com informações coletadas, foi o relacionado a disponibilidade $(\mu 1)$, sendo este o que retrata o tempo que o equipamento esteve em disposição para executar a operação em relação a quantidade programada. Para a unidade de tempo definida por minuto, tem-se:

Para o equipamento 01:

$\mu 1=(\mathrm{TPP}-\mathrm{TPnP}) / \mathrm{TPP}$

$\mu 1=(360-22) / 360$

$\mu 1=93,88 \%$

Para o equipamento 02 :

$\mu 1=($ TPP - TPnP $) / T P P$

$\mu 1=(360-45) / 360$

$\mu 1=87,50 \%$

Para o equipamento 03:

$\mu 1=(\mathrm{TPP}-\mathrm{TPnP}) / \mathrm{TPP}$

$\mu 1=(360-37) / 360$

$\mu 1=89,72 \%$

Logo, o índice de desempenho $(\mu 2)$, que associa o tempo de ciclo por peça e quantidade de itens produzidos, foi estipulado para o caso dos equipamentos selecionados, obtendo-se os valores:

Para o equipamento 01 :

$\mu 2$ = Tempo de produção total / Tempo real de operação

$\mu 2=422 / 450$

$\mu 2=93,77 \%$

Para o equipamento 02 :

$\mu 2=$ Tempo de produção total / Tempo real de operação

$\mu 2=379 / 450$

$\mu 2=84,22 \%$

Para o equipamento 03: 
$\mu 2$ = Tempo de produção total / Tempo real de operação

$\mu 2=365 / 450$

$\mu 2=81,11 \%$

Por fim, observou-se o índice referente à qualidade $(\mu 3)$, que engloba o número total de peças produzidas em conformidade, comparado com o número total de peças produzidas, sendo calculado por:

Para o equipamento 01:

$\mu 3=(\mathrm{QPP}-\mathrm{QPR}) / \mathrm{QPP}$

$\mu 3=(541-28) / 541$

$\mu 3=94,82 \%$

Para o equipamento 02 :

$\mu 3=(\mathrm{QPP}-\mathrm{QPR}) / \mathrm{QPP}$

$\mu 3=(541-33) / 541$

$\mu 3=93,90 \%$

Para o equipamento 03:

$\mu 3=(\mathrm{QPP}-\mathrm{QPR}) / \mathrm{QPP}$

$\mu 3=(541-25) / 541$

$\mu 3=95,34 \%$

Assim, com os índices calculados por cada equipamento, o valor da eficiência global (OEE) é obtido por meio do resultado do produto entre os três parâmetros, sendo este a partir da fórmula:

$\mathrm{OEE}=\mu$ geral $=\mu 1 \times \mu 2 \times \mu 3$

Os valores do OEE, por equipamento, são reunidos na Tabela 2.

Tabela 2 - Valor do OEE por equipamento

\begin{tabular}{cccccc}
\hline Equipamento & $\mu 1(\%)$ & $\mu 2(\%)$ & $\mu 3(\%)$ & OEE (\%) & Aceitabilidade \\
\hline 1 & 93,88 & 93,77 & 94,82 & 83,47 & Muito bom \\
2 & 87,50 & 84,22 & 93,90 & 69,19 & Bom \\
3 & 89,72 & 81,11 & 95,34 & 69,38 & Bom \\
Média & 90,37 & 86,37 & 94,69 & 74,01 & - \\
\hline
\end{tabular}

Fonte: Dados da pesquisa (2018)

Conforme estimado, os valores correspondentes aos equipamentos de números 2 e 3 , coincidentemente relativos às máquinas de envase ou engarrafamento, devem ser melhorados, tendo em vista que o aceitável seria acima ou próximo de 85 \%. Em suma, tem-se a representação a partir do Gráfico 1. 
Gráfico 1 - Dados obtidos por equipamento

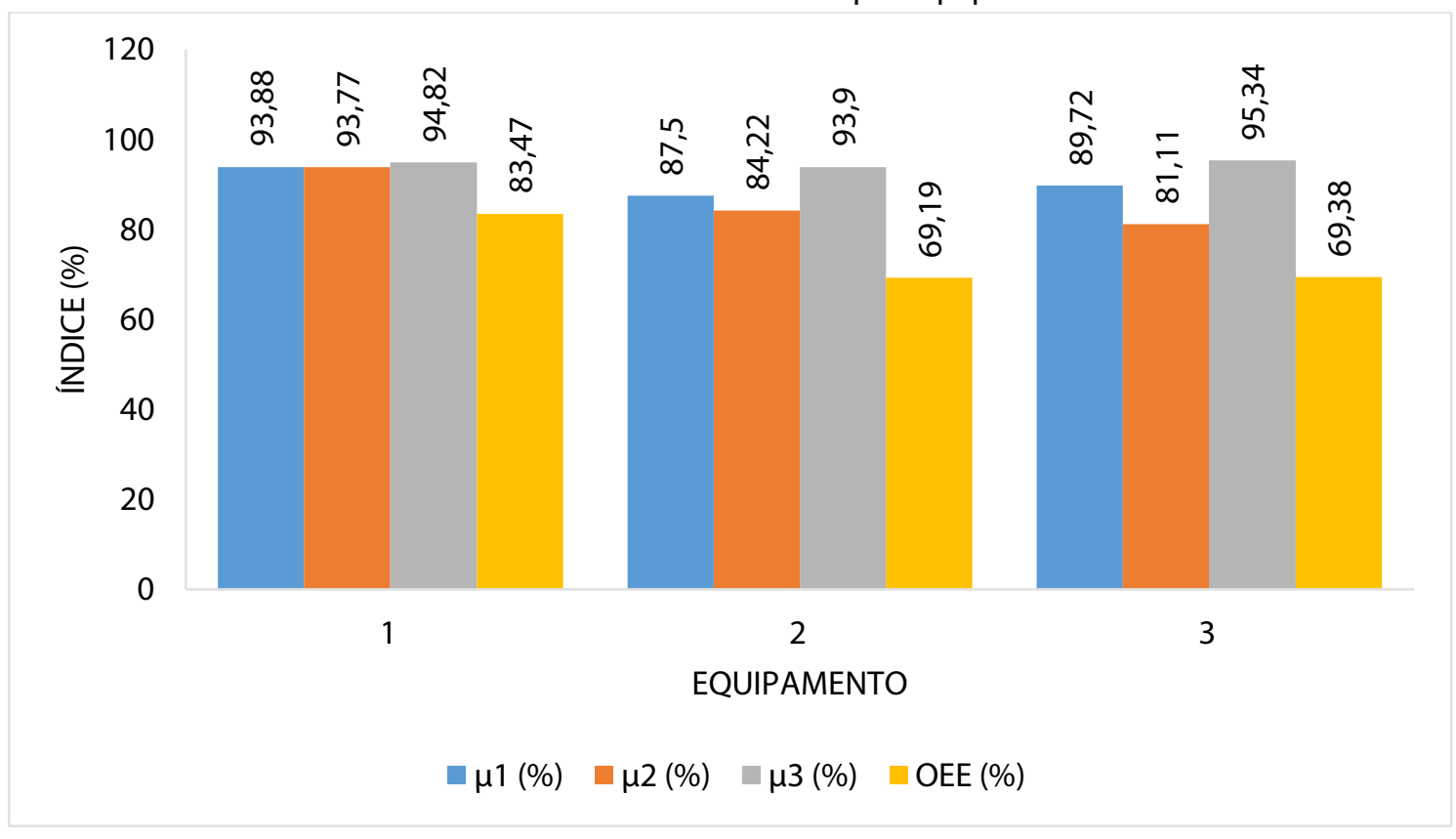

Fonte: Dados da pesquisa (2018).

Observa-se, a partir dos resultados obtidos e apresentados anteriormente, que a empresa ainda deve buscar melhorar os valores do OEE para os equipamentos analisados. Na indústria vinícola, a eficácia e eficiência do equipamento desempenha um papel importante para diminuir o número de desperdícios por falta de qualidade e ainda para alcançar maior produtividade, sendo, portanto, uma oportunidade para a empresa evoluir em termos de efetividade das suas operações.

Com os valores obtidos no OEE geral, pode-se notar e apontar a percentagem do tempo verdadeiramente produtivo, observando os três fatores; ou seja, disponibilidade, desempenho e qualidade. Assim, realizando essa análise de tempo com o OEE, tem-se a possibilidade de corrigir e eliminar o tempo perdido que pode ocorrer na fabricação de vinhos e outros produtos, na empresa estudada.

Ainda nesse sentido, o fator qualidade deve ser verificado com maior atenção. Esse índice diz respeito aos produtos que são fabricadas durante o processo de produção na vinícola e estão associados diretamente com a perda de qualidade. Isto é, apontam para o nível (\%) dos produtos que não atendem aos padrões e requisitos técnicos e apresentam também alguns defeitos, e que podem precisar de retrabalho para que atinjam os padrões aceitáveis pelo mercado. Portanto, para a empresa, esse valor do índice referente à qualidade ( $\mu 3)$, é uma informação relevante a ser considerada em análises futuras, como forma de comparar a evolução em termos de agregação de valor aos produtos da vinícola.

Ademais, tais considerações concordam com a afirmativa de Singh e Narwal (2017), onde os autores citam que a investigação do OEE fornece um processo sistemático para identificar facilmente fontes de perdas de produtividade para que seja possível, efetivamente, aplicar recursos para melhorar o processo de fabricação e o desempenho da empresa.

Assim, o plano de ação foi traçado para, com base nos fatores identificados que limitam os equipamentos, atividades sejam capazes de aumentar a eficiência dos mesmos.

\subsection{Plano de ação - 5W1H}

O Plano de Ação, estruturado no Quadro 3, é um documento que será utilizado para o planejamento de atividades que contribuirão para atingimento de um melhor resultado em termos de rendimento e eficiência dos equipamentos do processo produtivo da vinícola. 
Quadro 3 - Plano de ação - 5W1H

\begin{tabular}{|c|c|c|c|c|c|}
\hline O quê (What) & Por quê (Why) & $\begin{array}{l}\text { Onde } \\
\text { (Where) }\end{array}$ & $\begin{array}{l}\text { Quem } \\
\text { (Who) }\end{array}$ & $\begin{array}{l}\text { Quando } \\
\text { (When) }\end{array}$ & Como (How) \\
\hline $\begin{array}{c}\text { Estudos sobre } \\
\text { eficiência dos } \\
\text { equipamentos por } \\
\text { produto }\end{array}$ & $\begin{array}{c}\text { Identificar possíveis } \\
\text { gargalos }\end{array}$ & Produção & Gestão local & $\begin{array}{l}\text { Em até } 60 \\
\text { dias }\end{array}$ & $\begin{array}{c}\text { Cálculos de OEE } \\
\text { dos equipamentos }\end{array}$ \\
\hline $\begin{array}{c}\text { Analisar formas de } \\
\text { aproveitar melhor } \\
\text { as máquinas de } \\
\text { envase }\end{array}$ & $\begin{array}{c}\text { Otimizar a } \\
\text { produtividade }\end{array}$ & Produção & Gestão local & $\begin{array}{l}\text { Em até } 60 \\
\text { dias }\end{array}$ & $\begin{array}{l}\text { Estudo de } \\
\text { engenharia de } \\
\text { métodos }\end{array}$ \\
\hline $\begin{array}{c}\text { Estratificar e } \\
\text { monitorar tempo } \\
\text { gasto por } \\
\text { equipamento em } \\
\text { paradas não } \\
\text { planejadas } \\
\end{array}$ & $\begin{array}{l}\text { Aumentar o índice } \\
\text { de disponibilidade } \\
(\mu 1)\end{array}$ & Produção & Gestão local & $\begin{array}{l}\text { Em até } 40 \\
\text { dias }\end{array}$ & $\begin{array}{l}\text { Observação } \\
\text { registrada em } \\
\text { planilhas, por } \\
\text { equipamento }\end{array}$ \\
\hline $\begin{array}{l}\text { Implementar } \\
\text { gestão da } \\
\text { manutenção na } \\
\text { planta }\end{array}$ & $\begin{array}{c}\text { Aumentar o índice } \\
\text { de disponibilidade } \\
(\mu 1) \text { e } \\
\text { consequentemente } \\
\text { evitar paradas para } \\
\text { correção }\end{array}$ & Manutenção & Gestão local & $\begin{array}{l}\text { Em até } 40 \\
\text { dias }\end{array}$ & $\begin{array}{l}\text { Definição e } \\
\text { treinamento de } \\
\text { equipe de } \\
\text { manutenção }\end{array}$ \\
\hline $\begin{array}{l}\text { Analisar e detectar } \\
\text { perdas por falta de } \\
\text { qualificação de } \\
\text { funcionários }\end{array}$ & $\begin{array}{c}\text { Aumentar o índice } \\
\text { de desempenho }(\mu 2)\end{array}$ & Produção & Gestão local & $\begin{array}{l}\text { Em até } 40 \\
\text { dias }\end{array}$ & $\begin{array}{c}\text { Observação } \\
\text { estratificada por } \\
\text { funcionário versus } \\
\text { número de perdas } \\
\text { médio no período }\end{array}$ \\
\hline $\begin{array}{l}\text { Fazer campanha } \\
\text { sobre o mau uso } \\
\text { de equipamentos }\end{array}$ & $\begin{array}{c}\text { Aumentar o índice } \\
\text { de desempenho }(\mu 2)\end{array}$ & Produção & Gestão local & $\begin{array}{l}\text { Em até } 20 \\
\text { dias }\end{array}$ & $\begin{array}{c}\text { Treinamento } \\
\text { acerca do uso e } \\
\text { funcionalidade } \\
\text { dos } \\
\text { equipamentos. } \\
\text { Reciclagem sobre } \\
\text { operação de } \\
\text { máquinas. }\end{array}$ \\
\hline $\begin{array}{l}\text { Analisar impacto } \\
\text { do refugo no OEE }\end{array}$ & $\begin{array}{l}\text { Aumentar o índice } \\
\text { de qualidade }(\mu 3)\end{array}$ & Produção & Gestão local & $\begin{array}{l}\text { Em até } 30 \\
\text { dias }\end{array}$ & $\begin{array}{l}\text { Estratificar } \\
\text { quantidade de } \\
\text { refugo por tipo de } \\
\text { equipamento } \\
\text { e/ou operador } \\
\text { e/ou produto }\end{array}$ \\
\hline $\begin{array}{l}\text { Estipular custo } \\
\text { com retrabalho }\end{array}$ & $\begin{array}{l}\text { Aumentar o índice } \\
\text { de qualidade }(\mu 3)\end{array}$ & Produção & Gestão local & $\begin{array}{l}\text { Em até } 30 \\
\text { dias }\end{array}$ & $\begin{array}{l}\text { Estudo financeiro } \\
\text { aplicado }\end{array}$ \\
\hline $\begin{array}{c}\text { Elaborar plano de } \\
\text { manutenção } \\
\text { corretiva }\end{array}$ & $\begin{array}{l}\text { Corrigir falhas ao } \\
\text { longo do processo } \\
\text { que geram } \\
\text { desperdícios }\end{array}$ & Produção & Direção & $\begin{array}{c}\text { Em no } \\
\text { máximo } 25 \\
\text { dias }\end{array}$ & $\begin{array}{c}\text { Reparo nos } \\
\text { equipamentos do } \\
\text { processo }\end{array}$ \\
\hline $\begin{array}{l}\text { Implantação de } \\
\text { programa } 5 S\end{array}$ & $\begin{array}{c}\text { Promover a eficácia } \\
\text { do processo } \\
\text { produtivo }\end{array}$ & Produção & Direção & $\begin{array}{c}\text { Em no } \\
\text { máximo } 60 \\
\text { dias }\end{array}$ & $\begin{array}{c}\text { Garantir um } \\
\text { ambiente } \\
\text { organizado, limpo } \\
\text { e seguro }\end{array}$ \\
\hline
\end{tabular}

Fonte: Autoria própria (2018). 
Diante do exposto, o 5W1H poderá auxiliar a gestão da empresa na obtenção de melhor desempenho, pois traduz-se como um apoio na busca por qualidade total, devendo ser seguido e melhorado de acordo com as necessidades.

\section{CONSIDERAÇÕES FINAIS}

O processo produtivo do vinho apresenta-se como um dos mais antigos, com diversas particularidades, como o tipo de produto e a filosofia de cada produtor. Por outro lado, é um processo que, de modo geral, apresenta pouca intervenção humana, ou seja, conta atualmente com grande variedade de equipamentos e tecnologias capazes de adequação por empresa e/ou planta. Logo, a partir do presente estudo pôde-se perceber a importância de avaliar, constantemente o desempenho do processo com foco nos equipamentos, utilizando o OEE. É notória sua relevância no contexto industrial e sua aplicação pode auxiliar os gestores na tomada de decisão, otimizando os ganhos e a produtividade final.

A relação entre disponibilidade, desempenho e qualidade estabelecida no OEE é fundamental e útil para diversos contextos e pode ser empregado como um indicador relevante, que traduz a realidade das operações, nos processos de tomada de decisão das empresas. O índice mede, uniformemente e de forma consistente, os fatores que afetam diretamente o desempenho dos equipamentos e, respectivamente, das operações locais.

Com os cálculos de eficiência, é possível identificar se os resultados reais estão dentro do padrão desejado, no qual, quando visto que isto não ocorre, a intervenção deve ser feita. Quando constante o acompanhamento do indicador, as ações com base em dados atualizados podem ser tomadas, acarretando uma melhoria contínua da produção, reduzindo ainda despesas com manutenção e apontando gargalos com mais rapidez. Ademais, por ser um cálculo que depende de diversos fatores, o recomendado é que seja executado sempre de forma automática, viabilizando a agilidade.

No caso da empresa em estudo, pôde-se perceber que dos equipamentos escolhidos para análise, a prensa (OEE $=83,47 \%$ ) e as duas máquinas de envase $(69,19 \%$ e $69,38 \%$, respectivamente), podem ter seu desempenho melhorado. Para isto, o plano de ação será fundamental ter sua execução na totalidade para que a empresa tenha, de fato, mais lucro e melhor qualidade nos produtos fabricados.

Como principal limitação do estudo, pode-se citar a quantidade de equipamentos analisados, tendo em vista que a empresa conta com uma vasta combinação destes. Além disso, a verificação dos valores foi executada em apenas um mês, recomendando-se, portanto, para estudos futuros, a análise e comparação dos valores por mês. Assim, a pesquisa poderá ter uma base maior de discussão e notar a evolução da planta quanto ao rendimento de seus equipamentos e melhoria contínua do processo.

\section{REFERÊNCIAS}

ADESTA, E. Y.T.; PRABOWO, H. A.; AGUSMAN, D. Evaluating 8 pillars of Total Productive Maintenance (TPM) implementation and their contribution to manufacturing performance. IOP Conf. Series: Materials Science and Engineering, 2018.

AFEFY, I. H. Implementation of Total Productive Maintenance and Overall Equipment Effectiveness Evaluation, International Journal of Mechanical \& Mechatronics Engineering, v. 13, n. 1, p. 69-75, 2013.

AMAN, Z.; EZZINE, L.; FATTAH, J.; LACHHAB, A. Improving efficiency of a production line by Using Overall Equipment Effectiveness: A case study. INTERNATIONAL CONFERENCE ON INDUSTRIAL ENGINEERING AND OPERATIONS MANAGEMENT, 2017, Morocco. Proceedings [...]. Rabat, Morocco, 2017.

ANTUNES, J. et al. Sistemas de produção: conceitos e práticas para projeto e gestão da produção enxuta. Porto Alegre: Bookman, 2008. 
BARIANI, L.; DEL'ARCO JÚNIOR, A. P. Utilização da tecnologia da informação por grupos integrados de manufatura para o controle de indicadores de produção enxuta. Revista de Ciências Humanas, v. 12, n. 1, p. 67-79, 2006.

CHARAF, K.; DING, H. Is Overall Equipment Effectiveness (OEE) Universally Applicable? The Case of SaintGobain. International Journal of Economics and Finance. v. 7, n. 2, p. 241-252, 2015.

CHEN, C.; LU, I.; WANG, K.; JANG, J.; DAHLGAARD, J. Development of quality management in Taiwan the past, present and future. Total Quality Management Business \& Business Excellence, v. 26, n. 1-2, p. 3-13, 2015.

CHONG, M.; CHIN, J.; HAMZAH, H. Transfer of total productive maintenance to supply chain. Total Quality Management Business \& Business Excellence, v. 23, n. 3-4, p. 467-488. 2012.

CORRÊA, H. L.; CORRÊA, C. A. Administração de Produções e Operações. São Paulo: Atlas, 2004.

DAL, B.; TUGWELL, P.; GREATBANKS, R. Overall equipment effectiveness as a measure for operational improvement: a practical analysis, International Journal of Operations \& Production Management, v. 20, n. 12, p. $1488-502,2000$.

GARZA-REYES, J. A.; ELDRIDGE, S.; BARBER, K. D.; SORIANO-MEIER, H. Overall equipment effectiveness (OEE) and process capability (PC) measures: A relationship analysis, International Journal of Quality \& Reliability Management, v. 27, n. 1, p. $48-62,2010$.

GIL, A. C. Como elaborar projetos de pesquisa. 4. ed. São Paulo: Atlas, 2007.

GUPTA, A. K.; GARG, R. K. OEE Improvement by TPM Implementation: A Case Study. International Journal of IT Engineering and Applied Sciences Research (IJIEASR), v. 1, n. 1, p. 115-124, 2012.

HANSEN, R. C. Eficiência global dos equipamentos: uma poderosa ferramenta de produção/manutenção para o aumento dos lucros. Porto Alegre: Bookman, 2006.

JEONG, K-Y.; PHILLIPS, D.T. Operational efficiency and effectiveness measurement, International Journal of Operations \& Production Management, v. 21 n. 11, p. 1404-1416, 2001.

KARDEC, A.; NASCIF, J. Manutenção: função estratégica. 3. ed. Rio de Janeiro: Ed. Qualitymark, 2009.

KATKAMWAR, S. G.; WADATKAR, S. K.; PAROPATE, R. V. Study of Total Productive Maintenance \& Its Implementing Approach in Spinning Industries. International Journal of Engineering Trends and Technology (IJETT), v. 4, n. 5, p. 1750-1754, 2013.

KRYNKE, M.; KNOP, K.; MIELCZAREK, K. Analysis of the modernity and effectiveness of chosen machines in the processing of high-molecular materials. Production Engineering Archives, v. 3, n. 2, p. 18-21, 2014.

MADANHIRE, I.; MBOHWA, C. Implementing Successful Total Productive Maintenance (TPM) in a Manufacturing Plant. WORLD CONGRESS ON ENGINEERING, 2015, London, U.K. Proceedings [...]. London, UK : WCE, 2015.

MAIDEEN, N. C.; SAHUDIN, S.; MOHD YAHYA, N. H.; NORLIAWATI1, A. O. Practical Framework: Implementing OEE Method in Manufacturing Process Environment. IOP Conf. Series: Materials Science and Engineering, v. 114, p. 1-11, 2016.

MARKUS, P. R.; EBERHARD A. Enhancement of the overall equipment effectiveness measure: a contribution for handling uncertainty in shop floor optimization and production planning. International Journal of Industrial and Systems Engineering, v. 20, n. 2, p. 141-154, 2015.

MAŠIĆ, B.; DŽUNIĆ, M.; NEŠIĆ, S. Savremena teorija menadžmenta - škole i novi pristupi. Beograd: Data status, 2014.

MOURTZIS, D.; DOUKAS, M. Decentralized Manufacturing Systems Review: Challenges and Outlook, Logistics Research, v. 5, n. 3-4, p. 113-121, 2012. 
PANDEY, D. S.; RAUT, N. Implementing TPM by doing Root Cause Analysis of the Downtime losses. International journal of advanced research in science, engineering and technology, v. 3, n. 2, p. 13991405, 2016.

PARIKH, Y.; MAHAMUNI, P. Total Productive Maintenance: Need \& Framework. International Journal of Innovative Research in Advanced Engineering (IJIRAE), v. 2, n. 2, p. 126-130, 2015.

REEVES, M.; DEIMLER, M. Adaptability: The new competitive advantage. Harvard Business Review, v. 89, n. 78, p. 134-141. 2011.

SANTOS, P. V. S. A Indústria Vinícola no Vale do São Francisco e as Estratégias de Inserção no Mercado Nacional: Uma Revisão Bibliográfica. Future Studies Research Journal: Trends and Strategies, v. 9, n. 3, p. 39-69, 2017.

SANTOS, P. V. S. Aplicação do indicador Overall Equipment Effectiveness (OEE): um estudo de caso numa retífica e oficina mecânica. Brazilian Journal of Production Engineering, v. 4, n. 3, p. 1-18, 2018.

SANTOS, P. V. S.; ARAÚJO, M. A. Aplicação de ferramentas lean no setor de logística: um estudo de caso. Revista Gestão em Análise, v. 7, n. 2, p. 168-183, 2018.

SANTOS, P. V. S.; SANTOS, L. D. P. G. Dos.Gestão de indicadores: um estudo de caso no setor de serviços. Brazilian Journal of Production Engineering, v. 4, n. 4, p. 115-133. 2018.

SINGH, R.; SHAH, D. B.; GOHIL, A. M.; SHAH, M. H. Overall Equipment Effectiveness (OEE) Calculation Automation through Hardware \& Software Development, Procedia Engineering, v. 51, p. 579-584, 2013.

SINGH, M.; NARWAL, M. Measurement of Overall Equipment Effectiveness (OEE) of a manufacturing industry: An effective lean tool. International Journal of Recent Trends in Engineering and Research, v. 3, n. 5, p. 268-275, 2017.

SIVANANTHAM, A.; SIVARAM, N. M. A literature review on Total Productive Maintenance. International Journal of ChemTech Research, v. 10 n. 13, p. 138-144, 2017.

TAKAHASHI, Y; OSADA, T. Manutenção Produtiva Total. São Paulo: Instituto IMAN, 2000.

WUDHIKARN, R. A Framework for Integrating Overall Equipment Effectiveness with Analytic Network Process Method, International Journal of Innovation, Management and Technology, v. 4, n. 3, p. 351-355, 2013.

YIN, R. K. Estudo de caso - planejamento e métodos. 2. ed. Porto Alegre: Bookman. 2001.

YUNIAWAN, D.; ITO, T.; BIN, M. E. Calculation of Overall Equipment Effectiveness weight by Taguchi method with simulation. Concurrent Engineering, v. 21, n. 4, p. $296-306,2013$. 